Article

\title{
A Note on Symmetry of Birkhoff-James Orthogonality in Positive Cones of Locally $C^{*}$-algebras
}

\author{
Alexander A. Katz \\ Department of Mathematics and Computer Science, St. John's College of Liberal Arts and Sciences, \\ St. John's University, 8000 Utopia Parkway, SJH-334-G, Queens, NY 11439, USA; katza@stjohns.edu
}

Received: 19 May 2020; Accepted: 17 June 2020; Published: 23 June 2020

Abstract: In the present note some results of Kimuro, Saito, and Tanaka on symmetry of Birkhoff-James orthogonality in positive cones of $C^{*}$-algebras are extended to locally $C^{*}$-algebras.

Keywords: $C^{*}$-algebras; locally $C^{*}$-algebras; projective limit of projective family of $C^{*}$-algebras; locally Hilbert spaces; Birkhoff-James orthogonality; $A^{+}$-local left (right) symmetric point for Birkhoff-James orthogonality

AMS Classification: Primary 46K05, Secondary 46L89

\section{Introduction}

In 1935 Birkhoff introduced in [1], and in 1947 James studied in [2,3] a notion of orthogonality in Banach spaces in an attempt to generalize the standard notion of orthogonality in Hilbert spaces, which is important for study of Geometry of Banach spaces. More precisely, given a complex Banach space $X$ and two elements $x, y \in X$, one says that $x$ is Birkhoff-James orthogonal to $y$, denoted

$$
x \perp_{B} y,
$$

if

$$
\|x+\lambda y\| \geq\|x\|
$$

holds for all $\lambda \in \mathbb{C}$. This relation generalizes the standard notion of orthogonality in Hilbert spaces. However, for most Banach spaces it is not a symmetric relation, that is,

$$
x \perp_{B} y \text { does not imply } y \perp_{B} x
$$

in general.

A good overview of Birkhoff-James orthogonality for Banach spaces is given in 2012 by Alonso, Martini and $\mathrm{Wu}$ in their paper [4].

A special type of Banach spaces, and more specifically Banach algebras- the so called $C^{*}$-algebras, were introduced in 1943 by Gelfand and Naimark in their seminal paper [5]. At the present time the theory of $C^{*}$-algebras is well developed. For the development of the subject, see for example [6-8]. In $[9,10]$ one can find very important applications of $C^{*}$-algebras to Quantum Physics.

Recently, in 2019, Komuro, Saito and Tanaka in [11] studied elements in $C^{*}$-algebras on which Birkhoff-James orthogonality is symmetric in a suitable sense. More precisely, denoting by $A$ a $C^{*}$-algebra and by $A^{+}$its positive cone, the authors say that an element $a \in A^{+}$is an $A^{+}$-local left symmetric point for Birkhoff-James orthogonality if for all $b \in A^{+}$the relation

$$
a \perp_{B} b \text { implies } b \perp_{B} a .
$$


Analogously, $a \in A^{+}$is an $A^{+}$-local right symmetric point for Birkhoff-James orthogonality if for all $b \in A^{+}$the relation

$$
b \perp_{B} a \text { implies } a \perp_{B} b .
$$

Let $A$ be a $C^{*}$-algebra. The two main results of the aforementioned paper of Komuro, Saito and Tanaka characterize $A^{+}$-local left symmetric points and $A^{+}$-local right symmetric points in the following way:

Theorem 1 (Komuro-Saito-Tanaka). Let $A$ be $a C^{*}$-algebra and $a \in A^{+}$a positive element of norm one. Then $a$ is an $A^{+}$-local left symmetric point for Birkhoff-James orthogonality if and only if $a$ is a projection satisfying for each $b \in A^{+}$the property that there exists $\lambda \in \mathbb{C}$, such that

$$
a b a=\lambda a .
$$

Proof. See [11] for details.

Theorem 2 (Komuro-Saito-Tanaka). Let $A$ be a unital $C^{*}$-algebra and $a \in A^{+}$a non-zero positive element. Then $a$ is an $A^{+}$-local right symmetric point for Birkhoff-James orthogonality if and only if a is invertible.

Proof. See [11] for details.

The Hausdorff projective limits of projective families of Banach algebras as natural locally-convex generalizations of Banach algebras have been studied sporadically by many authors since 1952, when they were first introduced by Arens [12] and Michael [13]. The Hausdorff projective limits of projective families of $C^{*}$-algebras were first mentioned by Arens [12]. They have since been studied under various names by many authors. Development of the subject is reflected in the monograph of Fragoulopoulou [14]. We will follow Inoue [15] in the usage of the name locally $C^{*}$-algebras for these algebras. A lot of research has been done in the attempts to generalize to locally $C^{*}$-algebras the known results for $C^{*}$-algebras (see for example [15-19] to name a few). In particular, in [20] it has been shown how important it is in Physics to go out of category of $C^{*}$-algebras to the category of locally $C^{*}$-algebras- the reason is that not all operators of Quantum Physics, say for example the Energy operator, are bounded.

In the present notes we extend Theorems 1 and 2 of Komuro, Saito and Tanaka to locally $C^{*}$-algebras. This is the first attempt to study Geometry of Birkhoff-James orthogonality in locally convex spaces and algebras.

\section{Preliminaries}

First, let us recall some basic notions on topological *-algebras. A *-algebra (or involutory algebra) is a complex algebra $A$ with an involution

$$
\text { *: } A \rightarrow A,
$$

such that

$$
(a+\lambda b)^{*}=a^{*}+\bar{\lambda} b^{*}
$$

and

$$
(a b)^{*}=b^{*} a^{*}
$$

for every $a, b \in A$ and every $\lambda \in \mathbb{C}$.

A seminorm $\|$.$\| on A$ is a $C^{*}$-seminorm if it is submultiplicative, i.e.,

$$
\|a b\| \leq\|a\|\|b\|,
$$


and satisfies the $C^{*}$-condition, i.e.,

$$
\left\|a^{*} a\right\|=\|a\|^{2},
$$

for every $a, b \in A$. Note that the $C^{*}$-condition alone implies that $\|\cdot\|$ is submultiplicative, and in particular

$$
\left\|a^{*}\right\|=\|a\|,
$$

for every $a \in A$ (cf. for example [14]).

In the case when a seminorm $\|$.$\| on a { }^{*}$-algebra $A$ is a $C^{*}$-norm, and $A$ is complete in the topology generated by this norm, $A$ is called a $C^{*}$-algebra.

A topological ${ }^{*}$-algebra is a *-algebra $A$ equipped with a topology making the operations (addition, multiplication, additive inverse, involution) separately continuous. For a topological ${ }^{*}$-algebra $A$, we denote by $N(A)$ the set of continuous $C^{*}$-seminorms on $A$. If topology $\tau_{A}$ is generated by a separating and saturated family $N(A), A$ is called a locally $C^{*}$-algebra. One can see that in this case $N(A)$ is a directed set with respect to pointwise ordering, because

$$
\max \left\{\|\cdot\|_{\alpha},\|\cdot\|_{\beta}\right\} \in N(A)
$$

for every $\|\cdot\|_{\alpha},\|\cdot\|_{\beta} \in N(A)$, where $\alpha, \beta \in \Lambda$, with $\Lambda$ being a certain directed set.

For a topological ${ }^{*}$-algebra $A$, and $\|\cdot\|_{\alpha} \in N(A), \alpha \in \Lambda$,

$$
\operatorname{ker}\|\cdot\|_{\alpha}=\left\{a \in A:\|a\|_{\alpha}=0\right\}
$$

is a *-ideal in $A$, and $\|\cdot\|_{\alpha}$ induces a $C^{*}$-norm (we as well denote it by $\|\cdot\|_{\alpha}$ ) on the quotient algebra

$$
A_{\alpha}=A / \operatorname{ker}\|\cdot\|_{\alpha},
$$

and $A_{\alpha}$ is automatically complete in the topology generated by the norm $\|\cdot\|_{\alpha}$, thus is a $C^{*}$-algebra (see [14] for details). Each pair $\|\cdot\|_{\alpha},\|\cdot\|_{\beta} \in N(A)$, such that

$$
\beta \succeq \alpha,
$$

$\alpha, \beta \in \Lambda$, induces a natural (continuous) surjective *-homomorphism

$$
g_{\alpha}^{\beta}: A_{\beta} \rightarrow A_{\alpha},
$$

such that for any $\alpha, \beta, \gamma \in \Lambda$, such that

$$
\begin{gathered}
\gamma \succeq \beta \succeq \alpha, \\
g_{\alpha}^{\gamma}=g_{\alpha}^{\beta} \circ g_{\beta}^{\gamma} .
\end{gathered}
$$

Let now again, $\Lambda$ be a set of indices, directed by a relation (reflexive, transitive, antisymmetric) $" \preceq "$. Let

$$
\left\{A_{\alpha}, \alpha \in \Lambda\right\}
$$

be a family of $C^{*}$-algebras, and $g_{\alpha}^{\beta}$ be, for

$$
\alpha \preceq \beta,
$$

the continuous linear *-mappings

$$
g_{\alpha}^{\beta}: A_{\beta} \longrightarrow A_{\alpha} \text {, }
$$

so that

$$
g_{\alpha}^{\alpha}\left(x_{\alpha}\right)=x_{\alpha}
$$

for all $\alpha \in \Lambda$, and

$$
g_{\alpha}^{\beta} \circ g_{\beta}^{\gamma}=g_{\alpha}^{\gamma},
$$


whenever

$$
\alpha \preceq \beta \preceq \gamma .
$$

Let $\Gamma$ be the collections $\left\{g_{\alpha}^{\beta}\right\}$ of all such transformations. Let $A$ be a ${ }^{*}$-subalgebra of the direct product algebra

$$
\prod_{\alpha \in \Lambda} A_{\alpha}
$$

so that for its elements

$$
x_{\alpha}=g_{\alpha}^{\beta}\left(x_{\beta}\right),
$$

for all

$$
\alpha \preceq \beta,
$$

where

$$
x_{\alpha} \in A_{\alpha}
$$

and

$$
x_{\beta} \in A_{\beta} .
$$

Definition 1. The *-algebra A constructed above is called a Hausdorff projective limit of the projective family

$$
\left\{A_{\alpha}, \alpha \in \Lambda\right\},
$$

of $C^{*}$-algebras relatively to the collection

$$
\Gamma=\left\{g_{\alpha}^{\beta}: \alpha, \beta \in \Lambda: \alpha \preceq \beta\right\},
$$

and is denoted by

$$
\lim _{\alpha \in \Lambda} A_{\alpha}
$$

and is called the Arens-Michael decomposition of $A$.

It is well known (see, for example [21] or [22]) that for each $x \in A$, and each pair $\alpha, \beta \in \Lambda$, such that $\alpha \preceq \beta$, there is a natural projection

$$
\pi_{\beta}: A \longrightarrow A_{\beta}
$$

with the basic property

$$
\pi_{\alpha}(x)=g_{\alpha}^{\beta}\left(\pi_{\beta}(x)\right),
$$

and each projection $\pi_{\alpha}$ for all $\alpha \in \Lambda$ is continuous.

Theorem 3. A topological ${ }^{*}$-algebra $\left(A, \tau_{A}\right)$ over $\mathbb{C}$ is a locally $C^{*}$-algebra iff $A$ is a complete Hausdorff topological *-algebra in which the topology $\tau_{A}$ is generated by a saturated separating family $N(A)$ of $C^{*}$-seminorms.

Proof. See for example [14] for details.

Example 1. Every $C^{*}$-algebra is a locally $C^{*}$-algebra.

Example 2. A closed ${ }^{*}$-subalgebra of a locally $C^{*}$-algebra is a locally $C^{*}$-algebra.

Example 3. The product $\prod_{\alpha \in \Lambda} A_{\alpha}$ of $C^{*}$-algebras $A_{\alpha}$, with the product topology, is a locally $C^{*}$-algebra. 
Example 4. Let $X$ be a compactly generated Hausdorff space (this means that a subset $Y \subset X$ is closed iff $Y \cap K$ is closed for every compact subset $K \subset X)$. This spaces are called $k$-spaces in [23]. Then the algebra $C(X)$ of all continuous, not necessarily bounded complex-valued functions on $X$, with the topology of uniform convergence on compact subsets, is a locally $C^{*}$-algebra. It is well known that all metrizable spaces and all locally compact Hausdorff spaces are compactly generated (see [24] or [23] for details).

Let $A$ be a locally $C^{*}$-algebra. Then an element $a \in A$ is called bounded, if $\|a\|_{\infty}<\infty$, where

$$
\|a\|_{\infty}=\sup \left\{\|a\|_{\alpha}: \alpha \in \Lambda,\|\cdot\|_{\alpha} \in N(A)\right\} .
$$

The set of all bounded elements of $A$ is denoted by $b(A)$.

It is well-known that for each locally $C^{*}$-algebra $A$, its set $b(A)$ of bounded elements of $A$ is a locally $C^{*}$-subalgebra, which is a $C^{*}$-algebra in the norm $\|\cdot\|_{\infty}$, such that it is dense in $A$ in its topology $\tau_{A}$ (see for example [14]).

Let

$$
U_{\alpha}=\left\{a \in A:\|a\|_{\alpha} \leq 1\right\},
$$

$\alpha \in \Lambda$. Then $\left\{U_{\alpha}\right\}_{\alpha \in \Lambda}$ is a directed base with the associated family $\left\{\|\cdot\|_{\alpha}\right\}_{\alpha \in \Lambda}$ of $C^{*}$-seminorms on $A$ generating the topology $\tau_{A}$ of $A$.

Let $A^{*}$ as usual denote the conjugate space of $A$. For a given $\alpha \in \Lambda$,

$$
A^{*}(\alpha)=\left\{f \in A^{*}: f \text { is bounded on } U_{\alpha}\right\},
$$

denotes all functionals in $A^{*}$ that are bounded on $U_{\alpha}$. Let

$$
\Lambda(f)=\left\{\alpha \in \Lambda: f \text { is bounded on } U_{\alpha}\right\}
$$

denote all indices $\alpha$ in $\Lambda$ so that a given $f \in A^{*}$ is bounded on $U_{\alpha}$.

A linear functional on a locally convex space is continuous if and only if it is bounded on some neighborhood of zero. It means in our notation that $f \in A^{*}$ if and only if $\Lambda(f) \neq \varnothing$.

From that it follows that if $f \in A^{*}(\alpha)$, then there exists a linear functional $f_{\alpha}$ on $A_{\alpha}$, such that

$$
f(a)=f_{\alpha}\left(\pi_{\alpha}(a)\right)
$$

for all $a \in A$. Thus,

$$
A^{*}=\bigcup_{\alpha \in \Lambda} A^{*}(\alpha)
$$

Let now

$$
\begin{gathered}
A^{*}(+)=\left\{f \in A^{*}: f\left(a^{*} a\right) \geq 0 \text { for all } a \in A\right\}, \\
A^{*}(\alpha,+)=\left\{f \in A^{*}(\alpha): f\left(a^{*} a\right) \geq 0 \text { for all } a \in A\right\},
\end{gathered}
$$

$\alpha \in \Lambda$, and

$$
A_{\alpha}^{*}(+)=\left\{f_{\alpha} \in A_{\alpha}^{*}: f_{\alpha}\left(a_{\alpha}^{*} a_{\alpha}\right) \geq 0 \text { for all } a_{\alpha} \in A_{\alpha}\right\} .
$$

$\alpha \in \Lambda$.

One can see that:

(i). the mapping

$$
f \rightarrow f_{\alpha},
$$

$\alpha \in \Lambda$, is an algebraic isomorphism of $A^{*}(\alpha)$ onto $A_{\alpha}^{*}$;

(ii).

$$
\left\|f_{\alpha}\right\|=\sup _{a \in U_{\alpha}}|f(a)|,
$$


$a \in A, \alpha \in \Lambda$;

(iii). a functional $f \in A^{*}(\alpha)$ is positive (Hermitian) if and only iff $f_{\alpha} \in A_{\alpha}^{*}$ is positive (Hermitian);

(iv). if $A$ is unital, then the mapping

$$
f \rightarrow f_{\alpha},
$$

$\alpha \in \Lambda$, from $A^{*}(\alpha,+)$ onto $A_{\alpha}^{*}(+)$ is bi-continuous with respect to the relative $w^{*}$-topologies $\sigma\left(A^{*}, A\right)$ and $\sigma\left(A_{\alpha}^{*}, A_{\alpha}\right)$ of those spaces. Thus,

$$
A^{*}(+)=\bigcup_{\alpha \in \Lambda} A^{*}(\alpha,+)
$$

(see for example [15] for details.)

A non-zero positive functional $f$ on $A$ is called pure, it cannot be represented as a linear combination of two other positive functionals with non-negative coefficients. It is equivalent to a statement that from

$$
g \leq f,
$$

it follows that the exists $\lambda \in[0,1]$, so that

$$
g=\lambda f
$$

One can see that for $f \in A^{*}(\alpha,+)$ to be pure it is necessary and sufficient for $f_{\alpha}$ to be pure.

A family of Hilbert spaces family of Hilbert spaces $\left\{H_{\alpha}\right\}_{\alpha \in \Lambda}$ called inductive if for $\alpha, \beta \in \Lambda$, and

$$
\alpha \preceq \beta,
$$

the following conditions hold:

(i).

$$
H_{\alpha} \subset H_{\beta}
$$

(ii).

$$
(., .)_{\alpha}=(., .)_{\beta}
$$

on $H_{\alpha}$.

Let

$$
H=\bigcup_{\alpha \in \Lambda} H_{\alpha}
$$

be a union of an inductive family of Hilbert spaces $\left\{H_{\alpha}\right\}_{\alpha \in \Lambda}$.

We define a topology $\tau_{H}$ on $H$ as follows:

(i).

$$
X \subset H
$$

is a closed set in $H$;

if and only if

(ii).

$$
X=H,
$$

or

(iii). for some $\alpha \in \Lambda$,

$$
X \subset H_{\alpha},
$$

and $H$ is closed in $H_{\alpha}$.

With that topology $\tau_{H}, H$ is called a locally Hilbert space. It is shown in [15] that $\left(H, \tau_{H}\right)$ is a $T_{1}$ topological space.

Let for each $\alpha \in \Lambda$,

$$
P_{\alpha}: H \rightarrow H_{\alpha}
$$


be the orthogonal projection from $\mathrm{H}$ onto $\mathrm{H}_{\alpha}$, and for each pair of indices $\alpha, \beta \in \Lambda$, such that

$$
\begin{gathered}
\beta \succeq \alpha, \\
P_{\alpha}^{\beta}: H_{\beta} \rightarrow H_{\alpha},
\end{gathered}
$$

be the orthogonal projection from $H_{\beta}$ onto $H_{\alpha}$. One can see that

$$
P_{\alpha}=P_{\alpha}^{\beta} \circ P_{\beta}
$$

and for each $\alpha \in \Lambda$, and each vector $\xi \in H$, there exists a decomposition

$$
\xi=\xi^{\prime}+\eta,
$$

such that

$$
\left(\xi^{\prime}, \eta\right)=0,
$$

$\xi^{\prime} \in H_{\alpha}$, and $\eta$ belongs to the complement of $H_{\alpha}$ in $H$, i.e.,

$$
P_{\alpha}(\xi)=\xi^{\prime},
$$

and

$$
P_{\alpha}(\eta)=0_{H}
$$

(see [15] for details.)

Each locally Hilbert space $\left(H, \tau_{H}\right)$ is a pre-Hilbert space in a canonical way, and each pre-Hilbert space can be endowed with structure of a locally Hilbert space, and

$$
H \cong \underset{\alpha \in \Lambda}{\lim } H_{\alpha}
$$

where $\Lambda$ can be taken of the same cardinality as the set of all finite-dimensional Hilbert subspaces of $H$ ordered by inclusion.(see [25] for details).

Let $\left(A, \tau_{A}\right)$ be a locally $C^{*}$-algebra in which the topology $\tau_{A}$ generated by separating and saturated family $N(A)$ of $C^{*}$-seminorms $\left\{\|\cdot\|_{\alpha}\right\}_{\alpha \in \Lambda}$. Thus,

$$
A \cong \lim _{\alpha \in \Lambda} A_{\alpha}
$$

i.e., A is topologically ${ }^{*}$-isomorphic to the projective limit of the projective family of $C^{*}$-algebras $\left\{A_{\alpha}\right\}_{\alpha \in \Lambda}$, where for each $\alpha \in \Lambda, A_{\alpha}$ is isometrically ${ }^{*}$-isomorphic to the factor algebra

$$
A / \text { ker }\|\cdot\|_{\alpha}
$$

endowed with the factor norm. (see for example [14] for details). In this case we call $A$ a locally $C^{*}$-algebra of type $\Lambda$.

Remark 1. Generally speaking, their may be locally $C^{*}$-algebras of different types when $\Lambda$ is more than countable.

We say that a locally Hilbert space $\left(H, \tau_{H}\right)$ is of type $\Lambda$, if there exists an inductive family of its Hilbert subspaces generating it, i.e.,

$$
H \cong \underset{\alpha \in \Lambda}{\lim _{\alpha \in \Lambda}} H_{\alpha}
$$

Let $B\left(H_{\alpha}\right)$ for each $\alpha \in \Lambda$, be the $C^{*}$-algebra of all bounded linear operators on $H_{\alpha}$. From the fact that the family of Hilbert spaces $\left\{H_{\alpha}\right\}_{\alpha \in \Lambda}$, generates the locally Hilbert spaces $H$ of type $\Lambda$, it follows 
that it is inductive, and that the family of $C^{*}$-algebras $B\left(H_{\alpha}\right)$ is projective. We denote by $L(H)$ the projective limit

$$
L(H) \cong \lim _{\alpha \in \Lambda} B\left(H_{\alpha}\right)
$$

of that family $\left\{B\left(H_{\alpha}\right)\right\}_{\alpha \in \Lambda}$.

Let

$$
T: H \rightarrow H,
$$

be a linear operator on locally Hilbert space $H$ of type $\Lambda$. For each $\alpha \in \Lambda$, let

$$
T_{\alpha}(\xi)=T(\xi) \text { when } \xi \in H_{\alpha} .
$$

The operator $T$ is called coherent if and only if for every $\alpha, \beta \in \Lambda$, such that $\alpha \preccurlyeq \beta$,

$$
P_{\alpha}^{\beta} \circ T=T \circ P_{\alpha}^{\beta} .
$$

Lemma 1 (Inoue). A coherent linear operator $T$ on $H$ is continuous if and only if for each $\alpha \in \Lambda, T_{\alpha} \in B\left(H_{\alpha}\right)$.

Proof. See [15] for details.

As a corollary one gets that each coherent continuous linear operator on $H$ leaves $H_{\alpha}$ for each $\alpha \in \Lambda$ invariant, i.e., for each $\alpha \in \Lambda$,

$$
T\left(H_{\alpha}\right) \subset H_{\alpha}
$$

For that reason a linear coherent continuous linear operator $T$ on $H$ is called a locally bounded operator (see [26]). If we define

$$
\pi_{\alpha}: \lim _{\alpha \in \Lambda} B\left(H_{\alpha}\right) \rightarrow B\left(H_{\alpha}\right)
$$

as

$$
\pi_{\alpha}: T \rightarrow T_{\alpha}
$$

for each $\alpha \in \Lambda$, then $L(H)$ can be identified with the locally $C^{*}$-algebra of type $\Lambda$ of all locally bounded linear operators on the locally Hilbert space $H$ of type $\Lambda$.

Let $A$ and $B$ be locally $C^{*}$-algebras of the same type $\Lambda$ with respective Arens-Michael decompositions

$$
A \cong \lim _{\alpha \in \Lambda} A_{\alpha} \text { and } B \cong \varliminf_{\alpha \in \Lambda} B_{\alpha}
$$

We call a ${ }^{*}$-homomorphism

$$
\varphi: A \rightarrow B
$$

a locally ${ }^{*}$-homomorphism, if and only if for each $\alpha \in \Lambda$, there exits a *-homomor-phism of $C^{*}$-algebras $A_{\alpha}$ and $B_{\alpha}$,

$$
\varphi_{\alpha}: A_{\alpha} \rightarrow B_{\alpha},
$$

so that

$$
\pi_{\alpha}^{B} \circ \varphi=\varphi_{\alpha} \circ \pi_{\alpha}^{A},
$$

where $\pi_{\alpha}^{A}$ (resp. $\pi_{\alpha}^{B}$ ) is the natural projection from $A$ onto $A_{\alpha}$ (resp. the natural projection from $B$ onto $B_{\alpha}$ )

By a locally *-representation $\{A, \mu, H\}$ of a locally $C^{*}$-algebra $A$ of type $\Lambda$ on a locally Hilbert space $H$ of type $\Lambda$ we understand a locally ${ }^{*}$-homomorphism

$$
\mu: A \rightarrow L(H),
$$


from $A$ into the $C^{*}$-algebra $B(H)$ of bounded linear operators on some Hilbert space $H$.

Let

$$
A \cong \lim _{\alpha \in \Lambda} A_{\alpha}
$$

be a locally $C^{*}$-algebra of type $\Lambda$,

$$
\mu: A \rightarrow L(H)
$$

be a *-homomorphism from $A$ into $L(H)$, where

$$
H \cong \underset{\alpha \in \Lambda}{\lim _{\alpha \in \Lambda}} H_{\alpha}
$$

is a locally Hilbert space of type $\Lambda$. Then one can see that $\{A, \mu, H\}$ is a locally ${ }^{*}$-representation of the locally $C^{*}$-algebra $A$ of type $\Lambda$ if and only if for each $\alpha \in \Lambda,\left\{A_{\alpha}, \mu_{\alpha}, H_{\alpha}\right\}$ is a ${ }^{*}$-representation of the $C^{*}$-algebra $A_{\alpha}$ on the Hilbert space $H_{\alpha}$.

\section{Symmetry of Birkhoff-James Orthogonality in Positive Cones of Locally $C^{*}$-algebras}

Let $A$ be a locally $C^{*}$-algebra, and $a, b \in A$. We say that $a$ is Birkhoff-James orthogonal to $b$, denoted by

$$
a \perp_{B} b,
$$

if for each $\alpha \in \Lambda$, the inequality

$$
\|a+\lambda b\|_{\alpha} \geq\|a\|_{\alpha},
$$

holds for all $\lambda \in \mathbb{C}$. We say that if $A^{+}$is the positive cone of $A$, the element $a \in A^{+}$is an $A^{+}$-local left symmetric point for Birkhoff-James orthogonality if for all $b \in A^{+}$the relation

$$
a \perp_{B} b \text { implies } b \perp_{B} a .
$$

Analogously, $a \in A^{+}$is an $A^{+}$-local right symmetric point for Birkhoff-James orthogonality if for all $b \in A^{+}$the relation

$$
b \perp_{B} a \text { implies } a \perp_{B} b .
$$

The following three lemmata are valid:

Lemma 2. Let

$$
A \cong \lim _{\alpha \in \Lambda} A_{\alpha},
$$

be a locally $C^{*}$-algebra of type $\Lambda$, and $a, b \in$ A. Then

$$
a \perp_{B} b
$$

in $A$ if and only if for each $\alpha \in \Lambda$,

$$
a_{\alpha} \perp_{B} b_{\alpha}
$$

in $A_{\alpha}$.

Proof. First assume that

$$
a \perp_{B} b .
$$

It implies that for all $\alpha \in \Lambda$,

$$
\|a+\lambda b\|_{\alpha} \geq\|a\|_{\alpha},
$$


holds for all $\lambda \in \mathbb{C}$. For a given arbitrary $\alpha \in \Lambda$, we conclude that

$$
\left\|a_{\alpha}+\lambda b_{\alpha}\right\|_{A_{\alpha}}=\|a+\lambda b\|_{\alpha} \geq\|a\|_{\alpha}=\left\|a_{\alpha}\right\|_{A_{\alpha}},
$$

thus, due to the arbitrarity of $\alpha$, in each $A_{\alpha}$,

$$
a_{\alpha} \perp_{B} b_{\alpha} .
$$

Conversely, let us assume that for each $\alpha \in \Lambda$,

$$
a_{\alpha} \perp_{B} b_{\alpha}
$$

in $A_{\alpha}$. It means that for each $\alpha \in \Lambda$,

$$
\left\|a_{\alpha}+\lambda b_{\alpha}\right\|_{A_{\alpha}} \geq\left\|a_{\alpha}\right\|_{A_{\alpha}} .
$$

From the last inequality we get that for each $\alpha \in \Lambda$,

$$
\|a+\lambda b\|_{\alpha}=\left\|a_{\alpha}+\lambda b_{\alpha}\right\|_{A_{\alpha}} \geq\left\|a_{\alpha}\right\|_{A_{\alpha}}=\|a\|_{\alpha},
$$

thus

$$
a \perp_{B} b,
$$

which was required.

Lemma 3. Let

$$
A \cong \lim _{\alpha \in \Lambda} A_{\alpha}
$$

be a locally $C^{*}$-algebra of type $\Lambda$, and $a, b \in A$. Then for each $\alpha \in \Lambda$,

$$
\pi_{\alpha}^{A}\left(A^{+}\right)=A_{\alpha}^{+} .
$$

Proof. See [15] for details.

Lemma 4. Let $A$ be a locally $C^{*}$-algebra. Then $a \in A^{+}$is a projection if and only if for all $\alpha \in \Lambda, a_{\alpha}$ is $a$ projection in $A_{\alpha}^{+}$.

Proof. Let $a \in A^{+}$be a projection, i.e., $a^{2}=a$. Applying, for all $\alpha \in \Lambda$, the natural projection $\pi_{\alpha}^{A}$ to the last identity, we get

$$
a_{\alpha}^{2}=\pi_{\alpha}^{A}(a)^{2}=\pi_{\alpha}^{A}\left(a^{2}\right)=\pi_{\alpha}^{A}(a)=a_{\alpha},
$$

thus $a_{\alpha}^{2}=a_{\alpha}$, i.e., $a_{\alpha}$ is a projection in $A_{\alpha}^{+}$.

Conversely, let for all $\alpha \in \Lambda, a_{\alpha} \in A_{\alpha}^{+}$be such that $a_{\alpha}^{2}=a_{\alpha}$. Let us consider a family $\left\{a_{\alpha}\right\}_{\alpha \in \Lambda}$. This family is a projective family, i.e., there exists a unique $a \in A$, such that for each $\alpha \in \Lambda$,

$$
\pi_{\alpha}^{A}(a)=a_{\alpha} .
$$

Let us consider $a^{2} \in A^{+}$. For each $\alpha \in \Lambda$,

$$
\pi_{\alpha}^{A}\left(a^{2}\right)=\pi_{\alpha}^{A}(a)^{2}=a_{\alpha}^{2}=a_{\alpha}
$$

which implies that $a^{2}=a$. Indeed, let us on the contrary assume that $a^{2} \neq a$. It would mean that there exists at least one $\alpha_{0} \in \Lambda$, such that $a_{\alpha_{0}}^{2} \neq a_{\alpha_{0}}$. This contradiction completes the proof.

Now we are ready to prove a version of Theorem 1 for locally $C^{*}$-algebras. 
Theorem 4. Let $A$ be a locally $C^{*}$-algebra of type $\Lambda$ and $a \in A^{+}$a positive element of $A$, such that for each $\alpha \in \Lambda$,

$$
\|a\|_{\alpha}=1
$$

Then $a$ is an $A^{+}$-local left symmetric point for Birkhoff-James orthogonality if and only if a is a projection satisfying for each $b \in A^{+}$, the property that there exists $\lambda \in \mathbb{C}$, such that

$$
a b a=\lambda a
$$

Proof. First note, that since $b \in A^{+}$, if $a$ is a projection and $\lambda \in \mathbb{C}$, then

$$
(a b a)(\lambda a)=(\lambda a)(a b a)=\lambda a b a
$$

thus the elements $\lambda a$ and $a b a$ always commute, and therefore both belong to the maximal commutative locally $C^{*}$-subalgebra of $A$ that contains $a$.

Let $a \in A^{+}$be an $A^{+}$-local left symmetric point for Birkhoff-James orthogonality such that for each $\alpha \in \Lambda$,

$$
\|a\|_{\alpha}=1
$$

From Lemmas 2 and 3 it follows that for each $\alpha \in \Lambda, a_{\alpha} \in A_{\alpha}^{+}$is an $A_{\alpha}^{+}$-local left symmetric point for Birkhoff-James orthogonality in $A_{\alpha}$, and

$$
\left\|a_{\alpha}\right\|_{A_{\alpha}}=\|a\|_{\alpha}=1
$$

From Theorem 1 applicable to $A_{\alpha}$ it follows that $a_{\alpha}$ is a projection and for each $b_{\alpha} \in A_{\alpha}^{+}$, there exists $\lambda \in \mathbb{C}$, such that

$$
a_{\alpha} b_{\alpha} a_{\alpha}=\lambda a_{\alpha}
$$

where $b_{\alpha}$ without loss of generality can be taken in be arbitrary projective family $\left\{b_{\beta}\right\}_{\beta \in \Lambda}$. Indeed, let

$$
\alpha \preccurlyeq \beta,
$$

$\alpha, \beta \in \Lambda$, and

$$
a_{\alpha} b_{\alpha} a_{\alpha}=\lambda_{1} \cdot a_{\alpha}
$$

but

$$
a_{\beta} b_{\beta} a_{\beta}=\lambda_{2} \cdot a_{\beta}
$$

We get that

$$
\lambda_{1} \cdot a_{\alpha}=a_{\alpha} b_{\alpha} a_{\alpha}=g_{\alpha}^{\beta}\left(a_{\beta} b_{\beta} a_{\beta}\right)=g_{\alpha}^{\beta}\left(\lambda_{2} \cdot a_{\beta}\right)=\lambda_{2} \cdot a_{\alpha},
$$

and

$$
\lambda_{1}=\lambda_{2}
$$

thus $\lambda$ does not depend of $\alpha \in \Lambda$, as long as $b_{\alpha}$ is taken from a projective family $\left\{b_{\beta}\right\}_{\beta \in \Lambda}$.

Therefore, there exists a unique $b \in A$, such that for each $\alpha \in \Lambda$,

$$
\pi_{\alpha}^{A}(b)=b_{\alpha} \in A
$$

We show that

$$
a b a=\lambda a .
$$

Indeed, if we assume that

$$
a b a \neq \lambda a
$$


for any $\lambda \in \Lambda$, there will exist $\alpha_{0} \in \Lambda$, such that

$$
\pi_{\alpha_{0}}^{A}(a b a)=\pi_{\alpha_{0}}^{A}(a) \pi_{\alpha_{0}}^{A}(b) \pi_{\alpha_{0}}^{A}(a)=a_{\alpha_{0}} b_{\alpha_{0}} a_{\alpha_{0}} \neq \lambda a_{\alpha_{0}}=\pi_{\alpha_{0}}^{A}(\lambda a) .
$$

Contradiction proves what required.

Conversely, let $a \in A^{+}$be a projection satisfying for each $\alpha \in \Lambda$,

$$
\|a\|_{\alpha}=1
$$

such that for each $b \in A^{+}$, there exists $\lambda \in \mathbb{C}$, such that

$$
a b a=\lambda a .
$$

The later means that for each $\alpha \in \Lambda$,

$$
\pi_{\alpha}^{A}(a b a)=\pi_{\alpha}^{A}(a) \pi_{\alpha}^{A}(b) \pi_{\alpha}^{A}(a)=a_{\alpha} b_{\alpha} a_{\alpha}=\pi_{\alpha}^{A}(\lambda a)=\lambda a_{\alpha},
$$

i.e.,

$$
a_{\alpha} b_{\alpha} a_{\alpha}=\lambda a_{\alpha}
$$

for each $b_{\alpha} \in A_{\alpha}^{+}$, and $a_{\alpha}$ is a projection in $A_{\alpha}$ due to Lemma 4 .

Applying Theorem 1 to algebra $A_{\alpha}$ we get that $a_{\alpha} \in A_{\alpha}^{+}$is an $A_{\alpha}^{+}$-local left symmetric point for Birkhoff-James orthogonality in $A_{\alpha}$, for each $\alpha \in \Lambda$. Applying Lemma 2 we get that $a$ is an $A^{+}$-local left symmetric point for Birkhoff-James orthogonality, which was required.

In order to establish a version of Theorem 2 for locally $C^{*}$-algebras, we would need the following:

Lemma 5. Let $A$ be a unital locally $C^{*}$-algebra of type $\Lambda$. Then $a \in A$ is invertible if and only if for all $\alpha \in \Lambda$, $a_{\alpha}$ is invertible.

Proof. Let $a \in A$ be invertible, i.e., there exists a unique $b \in A$, such that

$$
a b=\mathbf{1}_{A} .
$$

Applying for each $\alpha \in \Lambda$, the natural projection $\pi_{\alpha}^{A}$, we get that

$$
\pi_{\alpha}^{A}(a b)=\pi_{\alpha}^{A}(a) \pi_{\alpha}^{A}(b)=a_{\alpha} b_{\alpha}=\pi_{\alpha}^{A}\left(\mathbf{1}_{A}\right)=\mathbf{1}_{A_{\alpha}},
$$

and $a_{\alpha}$ is invertible in $A_{\alpha}$.

Conversely, let $a \in A$ be such that for each $\alpha \in \Lambda, a_{\alpha}$ is invertible in $A_{\alpha}$, i.e., there exists a unique $b_{\alpha} \in A_{\alpha}$ for each $\alpha \in \Lambda$, such that

$$
a_{\alpha} b_{\alpha}=\mathbf{1}_{A_{\alpha}} .
$$

Let $B$ be maximal commutative locally $C^{*}$-subalgebra of $A$, such that $a \in B$. It is obvious that $B$ is of the same type $\Lambda$, and $a_{\alpha}, b_{\alpha} \in B_{\alpha}$ for all $\alpha \in \Lambda$, and

$$
B \cong \varliminf_{\alpha \in \Lambda} B_{\alpha}
$$

where each $B_{\alpha}$ is a commutative unital $C^{*}$-subalgebra of $A_{\alpha}$. Using a Gelfand-Naimark type theorem for commutative locally $C^{*}$-algebras (see [27]) we get a functional locally $C^{*}$-algebra $C(X)$ of type $\Lambda$ of all continuous complex-valued functions on compactly generated completely regular topological space $X$ with generating family of Hausdorff compacts $X_{\alpha}$, such that

$$
B \cong C(X),
$$


for each $\alpha \in \Lambda$, and

$$
B_{\alpha} \cong C\left(X_{\alpha}\right),
$$

where $C\left(X_{\alpha}\right)$ is a commutative unital $C^{*}$-algebra under supremum norm. Let $f_{a} \in C(X)$, be the function corresponding to the element $a \in A$, and let $f_{a_{\alpha}} \in C\left(X_{\alpha}\right)$ be the function corresponding to $a_{\alpha}$. Since each $a_{\alpha}$ is invertible in $A_{\alpha}, f_{a_{\alpha}}$ is never equal to 0 for all $x_{\alpha} \in X_{\alpha}$, for each $\alpha \in \Lambda$. Thus $f_{a}$ is never equal to 0 for all $x \in X$, thus, there exists a function

$$
\frac{1}{f_{a}} \in C(X)
$$

Let $b$ be an element in $B$ corresponding to the function $\frac{1}{f_{a}}$. We get that

$$
a b=\mathbf{1}_{B}=\mathbf{1}_{A},
$$

thus, $a$ is invertible, and for each $\alpha \in \Lambda$,

$$
\pi_{\alpha}^{A}(b)=b_{\alpha}
$$

due to uniqueness of $b_{\alpha}$.

Now we are ready to prove a version of Theorem 2 for locally $C^{*}$-algebras.

Theorem 5. Let $A$ be a unital locally $C^{*}$-algebra and $a \in A^{+}$a non-zero positive element. Then $a$ is an $A^{+}$-local right symmetric point for Birkhoff-James orthogonality if and only if a is invertible.

Proof. Let $a$ be an $A^{+}$-local right symmetric point for Birkhoff-James orthogonality in $A$. Due to Lemma 2, for each $\alpha \in \Lambda$, each

$$
a_{\alpha}=\pi_{\alpha}^{A}(a)
$$

is an $A_{\alpha}^{+}$-local right symmetric point for Birkhoff-James orthogonality in $A_{\alpha}$. Applying Theorem 2 to the algebra $A_{\alpha}$, we get that each $a_{\alpha}$ is invertible, and from Lemma 5 it follows that $a$ is invertible in $A$.

Conversely, let $a \in A^{+}$be non-zero positive invertible element. From Lemma 3 and Lemma 5 it follows that each $a_{\alpha}$ is a non-zero positive invertible element in $A_{\alpha}^{+}$. Applying Theorem 2 to the algebra $A_{\alpha}$, we get that each $a_{\alpha}$ is an $A^{+}$-local right symmetric point for Birkhoff-James orthogonality in $A_{\alpha}$. From Lemma 2 it now follows that $a$ is an $A^{+}$-local right symmetric point for Birkhoff-James orthogonality in $A$.

Funding: This research received no external funding.

Conflicts of Interest: The author declares no conflict of interest.

\section{References}

1. Birkhoff, G. Orthogonality in linear metric spaces. Duke Math. J. 1935, 1, 169-172. (In English) [CrossRef]

2. James, R.C. Orthogonality and linear functionals in normed linear spaces. Trans. Amer. Math. Soc. 1947, 61, 265-292. (In English) [CrossRef]

3. James, R.C. Inner product in normed linear spaces. Bull. Am. Math. Soc. 1947, 53, 559-566. (In English) [CrossRef]

4. Alonso, J.; Martini, H.; Wu, S. On Birkhoff orthogonality and isosceles orthogonality in normed linear spaces. Aequ. Math. 2012, 83, 153-189. (In English) [CrossRef]

5. Gelfand, I.M.; Naimark, M.A. On the imbedding of normed rings into the ring of operators in Hilbert space. Rec. Math. [Mat. Sbornik] N.S. 1943, 12, 197-213. (In English)

6. Dixmier, J. C*-algebras. In Translated from the French by Francis Jellett; North-Holland Mathematical Library; North-Holland Publishing Co.: Amsterdam, The Netherlands; New York, NY, USA; Oxford, UK, 1977; Volume 15, 492p. (In English) 
7. Murphy, G.J. C*-algebras and Operator Theory; Academic Press, Inc.: Boston, MA, USA, 1990; 286p. (In English)

8. Pedersen, G.K. C*-algebras and Their Automorphism Groups, 2nd ed.; Edited and with a preface by Søren Eilers and Dorte Olesen; Pure and Applied Mathematics; Academic Press: Amsterdam, The Netherlands; London, UK, 2018; 520p. (In English)

9. Emch, G.G. Emch, G.G. Algebraic Methods in Statistical Mechanics and Quantum Field Theory; Interscience Monographs and Texts in Physics and Astronomy; John Wiley \& Sons, Inc.: New York, NY, USA; London, UK; Sydney, Australia; Toronto, ON, Canada, 1972; Volume XXVI, 333p. (In English)

10. Emch, G.G. Mathematical and Conceptual Foundations of 20th-Century Physics; North-Holland Mathematics Studies; North-Holland Publishing Co.: Amsterdam, The Netherlands, 1984; Volume 100, 549p. (In English)

11. Komuro, N.; Saito, K.-S.; Tanaka, R. On symmetry of Birkhoff orthogonality in the positive cones of $C^{*}$-algebras with applications. J. Math. Anal. Appl. 2019, 474, 1488-1497. (In English) [CrossRef]

12. Arens, R.F. A generalization of normed rings. Pac. J. Math. 1952, 2, 455-471. (In English) [CrossRef]

13. Michael, E.A. Locally Multiplicatively-Convex Topological Algebras; American Mathematical Society: Providence, RI, USA, 1952; 79p. (In English)

14. Fragoulopoulou, M. Topological Algebras with Involution; North-Holland Mathematics Studies; Elsevier Science B.V.: Amsterdam, The Netherlands, 2005; Volume 200, 495p. (In English)

15. Inoue, A. Locally $C^{*}$-algebra. Mem. Fac. Sci. Kyushu Univ. Ser. A 1971, 25, 197-235. (In English) [CrossRef]

16. Katz, A.A. A note on Civin-Yood Theorem for locally $C^{*}$-algebras. Malaya J. Mat. 2015, 3, 182-186. (In English)

17. Katz, A.A. A note on Jacobson-Rickart-Kadison theorem for locally $C^{*}$-algebras. Indian J. Math. 2016, 58. (In English); Seventh Dr. George Bachman Memorial Conference, suppl. (2019), pp. 1-7.

18. Phillips, N.C. Inverse limits of $C^{*}$-algebras. J. Oper. Theory 1988, 19, 159-195. (In English)

19. Schmüdgen, K. Uber LMC*-Algebren. Math. Nachr. 1975, 68, 167-182. (In German) [CrossRef]

20. Iguri, S.; Castagnino, M. The formulation of Quantum Mechanics in terms of nuclear algebras. Int. J. Theor. Phys. 1999, 38, 143-164. (In English) [CrossRef]

21. Trèves, F. Topological Vector Spaces: Distributions and Kernels; Academic Press: New York, NY, USA; London, UK, 1967; 565p. (In English)

22. Schaefer, H.H.; Wolff, M.P. Topological Vector Spaces, 2nd ed.; Graduate Texts in Mathematics; Springer: New York, NY, USA, 1999; Volume 3, 346p. (In English)

23. Engelking, R. General Topology, 2nd ed.; Translated from the Polish by the author; Sigma Series in Pure Mathematics; Heldermann Verlag: Berlin, Germany, 1989; Volume 6, 529p. (In English)

24. Kelley, J.L. General Topology; Reprint of the 1955 edition [Van Nostrand, Toronto, Ont.]; Graduate Texts in Mathematics, No. 27; Springer: New York, NY, USA; Berlin, Germany, 1975; 298p. (In English)

25. Gheondea, A. On locally Hilbert spaces. Opusc. Math. 2016, 36, 735-747. (In English) [CrossRef]

26. Gheondea, A. Operator models for Hilbert locally $C^{*}$-modules. Oper. Matrices 2017, 11, 639-667. (In English) [CrossRef]

27. Friedman, O.; Katz, A.A. On Gelfand-Naimark type theorems for unital abelian complex and real locally $C^{*}$-, and locally JB-algebras. In Topological Algebras and Their Applications; Walter de Gruyter GmbH \& Co KG: Berlin, Germany, 2018; pp. 73-108. (In English)

(C) 2020 by the authors. Licensee MDPI, Basel, Switzerland. This article is an open access article distributed under the terms and conditions of the Creative Commons Attribution (CC BY) license (http://creativecommons.org/licenses/by/4.0/). 